\title{
Identificação do lado de preferência mastigatória através de exame eletromiográfico comparado ao visual
}

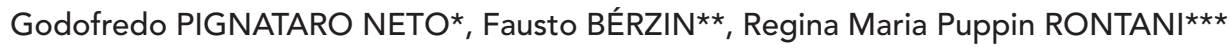

\begin{abstract}
Resumo
O objetivo deste estudo foi verificar a freqüência da mastigação preferencialmente unilateral através da eletromiografia comparada à inspeção visual. Participaram deste estudo 29 indivíduos saudáveis, alunos do Curso de Odontologia - Uniararas, na faixa etária entre 18 e 25 anos, de ambos os gêneros, selecionados através dos critérios: dentição permanente completa ou apenas ausência dos $3^{\text {os }}$ molares, sem sinais clínicos de disfunção craniomandibular ou doença periodontal. A análise eletromiográfica foi realizada nos músculos masseteres durante mastigação habitual de cenoura e parafilme. Considerou-se a presença de um lado de preferência mastigatória (LPM) quando os valores de amplitude de contração muscular obtidos em RMS (Root Mean Square) ou Valor Eficaz tivessem uma diferença de pelo menos 20\% entre os músculos masseteres direito e esquerdo. A inspeção visual foi realizada por um único observador durante as aquisições eletromiográficas. Considerou-se a presença de um lado de preferência mastigatória quando a mastigação ocorria com uma freqüência de ciclos mastigatórios pelo menos 30\% maior por um dos lados, após o registro de 20 ciclos mastigatórios consecutivos. Do total da amostra, 82,8\% (24/29) e 72,4\% (21/29) apresentaram um lado de preferência mastigatória durante a mastigação de cenoura e parafilme, respectivamente. A concordância entre a eletromiografia e a inspeção visual foi verificada em $83 \%$ das observações. Pode-se concluir que a maioria dos sujeitos examinados nessa pesquisa possuía um lado de preferência mastigatória durante mastigação habitual de cenoura e parafilme e que a eletromiografia pode ser usada como parâmetro para detecção do lado de preferência mastigatória.
\end{abstract}

Palavras-chave: Mastigação. Lado de preferência mastigatória. Mastigação unilateral. Eletromiografia.

\section{INTRODUÇÃO}

Uma das condições da estabilidade oclusal é mastigar dos dois lados, um de cada vez e protruir durante a incisão ${ }^{18,21}$. Além de manter o equilíbrio oclusal, a mastigação bilateral alternada, com excursões amplas e contatos oclusais fisiológicos, atividade muscular bilateralmente sincrônica e força uniforme sobre os tecidos de suporte dos dentes

* Mestre e Doutorando em Odontologia, Área de Fisiologia Oral pela FOP-UNICAMP; Especialista em Ortopedia Funcional dos Maxilares CFO-25/2002; Especialista em Dor Orofacial e Distúrbios Crâniomandibulares CFO-25/2002.

** Professor Doutor Titular da Área de Anatomia do Departamento de Morfologia da Faculdade de Odontologia de Piracicaba - UNICAMP.

*** Professora Doutora Associada da Área de Odontopediatria do Departamento de Odontologia Infantil da Faculdade de Odontologia de Piracicaba - UNICAMP. 
fornecem estímulos adequados para o desenvolvimento normal sagital e transversal da mandíbula e maxila além de participar direta e indiretamente na prevenção dos problemas periodontais e disfunções temporomandibulares ${ }^{18}$.

A mastigação bilateral alternada pode ser verificada com alta prevalência no homem silvícola ou agreste onde a mastigação ocorre com amplos movimentos de lateralidade mandibular, com a utilização de alimentos duros e secos que proporcionam estímulo funcional adequado para desenvolvimento dos arcos dentários e de todo o sistema mastigatório ${ }^{1,2,3,23}$.

Contudo o homem civilizado (em torno de $78 \%$ ) tende a possuir um lado de preferência mastigatória ${ }^{15}$. Considera-se a presença de um lado preferencial de mastigação quando o número de ciclos de um lado ocorre numa freqüência 30\% maior em relação ao número de ciclos realizados no lado oposto ${ }^{12}$.

Embora a mastigação possa ser realizada com movimentos unilaterais, isto não constitui uma função oclusal ideal. A mastigação preferencialmente unilateral promove estímulos diferenciados entre o lado de trabalho e o lado de não trabalho, causando o desenvolvimento desarmônico do esqueleto facial ${ }^{16,17}$.

Após a revolução industrial (últimos150-300 anos) os alimentos se tornaram pobres em textura não promovendo o esforço muscular necessário para a troca automática do lado de mastigação ${ }^{8}$.

Além do impedimento funcional ocasionado por perdas dentárias, presença de dor ou outras disfunções, o posicionamento dentário e os contatos oclusais são fatores determinantes da presença de um lado de preferência mastigatória - LPM ${ }^{5}$.

Planas ${ }^{14}$, após anos de observação clínica, verificou que o aumento da dimensão vertical durante os movimentos de lateralidade mandibular para a direita e esquerda forma um ângulo com o plano horizontal denominado de ângulo funcional mastigatório Planas (AFMP). Segundo Planas se os AFMPs forem diferentes (direito e esquerdo), a mastigação se torna preferencialmente unilateral do lado com AFMP menor. Recente estudo clínico identificou a presença de associação significante entre o lado de preferência mastigatória com o lado onde o AFMP é menor ${ }^{13}$.

Para a determinação do lado de preferência mastigatória, pode-se utilizar vários métodos diretos (observação visual) ou indiretos (cinegrafia, cinesiografia computadorizada), contudo a eletromiografia pode determinar o LPM com bastante confiabilidade 4 , pois os níveis de atividade mioelétrica são maiores no masseter ipsilateral quando comparados com a atividade massetérica contralateral ${ }^{10,11,19}$.

O objetivo deste estudo foi verificar a freqüência da mastigação preferencialmente unilateral através da eletromiografia dos músculos masseteres durante mastigação habitual de cenoura e parafilme.

\section{MATERIAL E MÉTODOS \\ Amostra}

Participaram deste estudo 29 indivíduos saudáveis, alunos do Curso de Graduação da Faculdade de Odontologia da Uniararas - FHO, na faixa etária entre 18 e 25 anos, de ambos os gêneros, selecionados através do seguinte critério: dentição permanente completa ou apenas ausência dos 3 os molares, sem sinais clínicos de disfunções craniomandibulares ou doença periodontal. Foi realizado exame clínico dos arcos dentários, tecidos moles e palpação dos músculos mastigatórios e articulação temporomandilar. O exame funcional consistiu na observação dos movimentos mandibulares sendo observados os movimentos de lateralidade direita e esquerda e protrusiva. Os dados anamnésicos pertinentes ao exame foram anotados juntamente com os dados do exame clínico-funcional. Todos os sujeitos da pesquisa participaram do estudo após fornecimento de termo de consentimento livre e esclarecido, previamente aprovado pelo Comitê de Ética em Pesquisas com Seres Humanos da Faculdade de Odontologia de Piracicaba através de protocolo n. 56/98. 


\section{VERIFICAÇÃO DO LADO DE PREFERÊNCIA MASTIGATÓRIA (LPM) Eletromiografia}

Os pacientes foram submetidos à análise eletromiográfica dos músculos masseteres para verificação do lado de preferência mastigatória. O masseter foi utilizado como referência principal para determinação do lado de preferência mastigatória como na metodologia descrita por Christensen e Radue ${ }^{4}$, já que o mesmo é um músculo de potência muscular, ao contrário do temporal, considerado como músculo posicionador da mandíbula.

Os registros foram realizados seguindo o protocolo experimental: os sujeitos da pesquisa foram colocados na posição sentada em altura adequada a cada um, com os pés apoiados sobre o solo e mãos relaxadas sobre as pernas, plano de Frankfurt paralelo ao solo e costas bem apoiadas.

Realizou-se três registros consecutivos durante a mastigação de cenoura, que foi utilizada por ser um alimento com bom grau de textura e dureza. A cenoura foi padronizada em quadrados de $2,5 \mathrm{~cm}^{2} / 3 \mathrm{~mm}$ (Fig. 1).

Os sujeitos mastigaram de forma livre sem indução para que se pudesse registrar a mastigação habitual que se caracteriza por ser inconsciente e controlada por regiões extrapiramidais do sistema nervoso motor.

Os registros foram realizados durante o tempo de 15 segundos. Posteriormente eliminou-se os 5 segundos iniciais correspondentes à fase inicial da mastigação (fase de trituração).

Foram realizados, seguindo-se o mesmo protocolo, os registros durante a mastigação de parafilme (lâmina de parafina com $5 \mathrm{~cm}^{2}$, dobrada duas vezes, ficando com tamanho de $2,5 \mathrm{~cm}^{2}$ ). O parafilme foi utilizado devido à sua ampla utilização em experimentos desta natureza (Fig. 1).

Foi utilizado um eletromiógrafo Lynx com sistema de Aquisição de Dados - placa CAD-12/32Lynx (Fig. 2B), além de programas de tratamento e leitura de dados Aqdados-Lynx DOS/Windows, instalado em computador de mesa (Fig. 2C).

Foram utilizados eletrodos ativos de superfície (Fig. 2A) com as seguintes características: (material: $\mathrm{AgCl}$, forma: retangular $(2,6 \mathrm{~cm} \times 1,8 \mathrm{~cm}$.), ganho de sinal: 20; distância dos pólos: $1 \mathrm{~cm}$.). Localização: colocado transversalmente sobre o ventre do músculo masseter (perpendicularmente às suas fibras) conforme a figura 3 , preso com esparadrapo (micropore) após a prévia limpeza da pele com álcool.

Detecção eletromiográfica: diferencial. Impedância de entrada: 10 M. CMMR: $>90$ db. SNR: comum. Filtros: Butterworth (altas: $500 \mathrm{~Hz}$ baixas: $20 \mathrm{~Hz}$ ).

Para se determinar o LPM utilizou-se os valores de amplitude de contração muscular durante os 10 segundos finais de mastigação habitual de cenoura e parafilme, dados obtidos diretamente pelo software em RMS (Root Mean Square) ou Valor Eficaz. O valor do RMS foi obtido a partir do sinal eletromiográfico considerado (dado na mesma unidade da grandeza do sinal) fornecido diretamente pelo programa utilizado (Fig.4).

Calculou-se a média aritmética das três aquisições de cenoura e parafilme para maior confiabilidade de resultados.

Cabe salientar que os dados eletromiográficos não foram normalizados, pois se almejava os valores de amplitude muscular, sendo comparados os masséteres direito e esquerdo do mesmo sujeito da pesquisa em uma única sessão e sob as mesmas condições experimentais (sem alteração no posicionamento dos eletrodos, posição postural dos sujeitos da pesquisa e condições ambientais como temperatura, horário, etc.).

Considerou-se a presença de um lado de preferência mastigatória quando os valores de RMS obtidos de um lado fossem pelo menos 20\% maiores que do outro lado. Este percentual foi obtido a partir da média dos valores obtidos no estudo, ou seja, os valores obtidos em RMS foram em média 20\% maiores de um lado em relação ao outro. 


\section{Observação Visual}

Foi utilizada a observação visual, realizada durante o exame eletromiográfico para verificar a coincidência entre a observação visual e o re- sultado dos registros eletromiográficos realizados durante a mastigação habitual de cenoura e parafilme. A observação visual foi realizada de forma direta observando-se a contração muscular e a di-

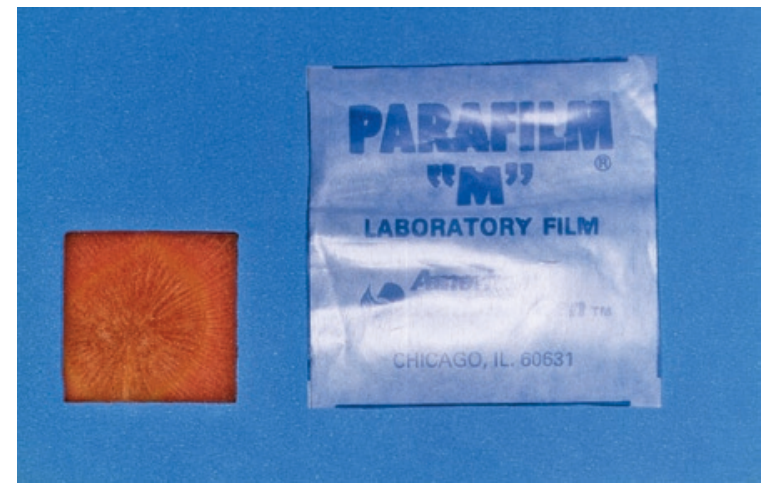

FIGURA 1 - Cenoura (padronizada em quadrados de $2,5 \mathrm{~cm}^{2} / 3 \mathrm{~mm}$ ) e Parafilme (lâmina de parafina com $5 \mathrm{~cm}^{2}$ ) utilizados para a obtenção dos registros eletromiográficos durante a mastigação habitual.

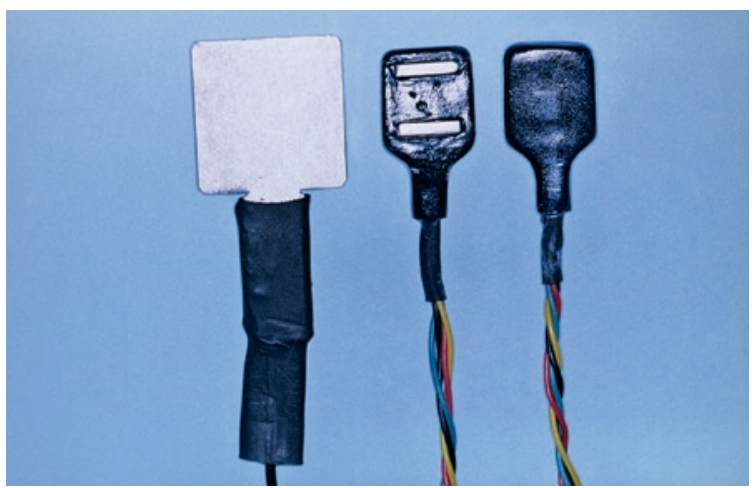

FIGURA 2 - Eletrodo ativo de superfície com as seguintes características: (material: $\mathrm{AgCl}$, forma: retangular $(2,6 \mathrm{~cm} \times 1.8 \mathrm{~cm}$.), ganho de sinal: 20; distância dos pólos: $1 \mathrm{~cm}$.)

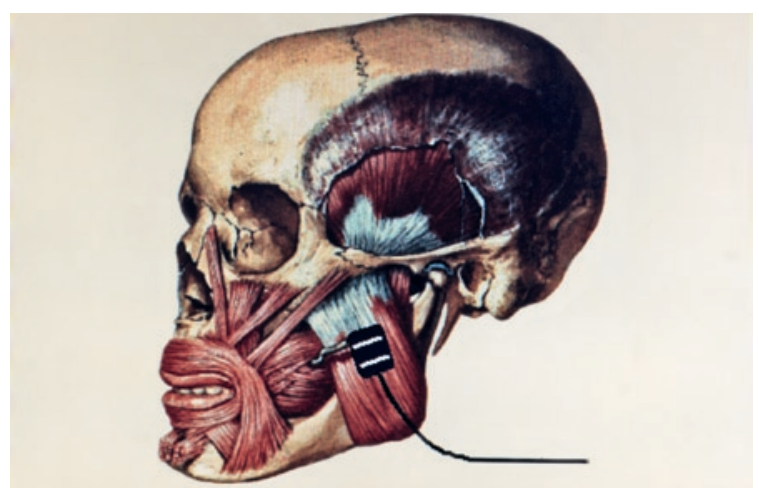

FIGURA 3 - Eletrodo ativo de superfície colocado transversalmente sobre 0 ventre do músculo masseter, perpendicularmente às suas fibras.

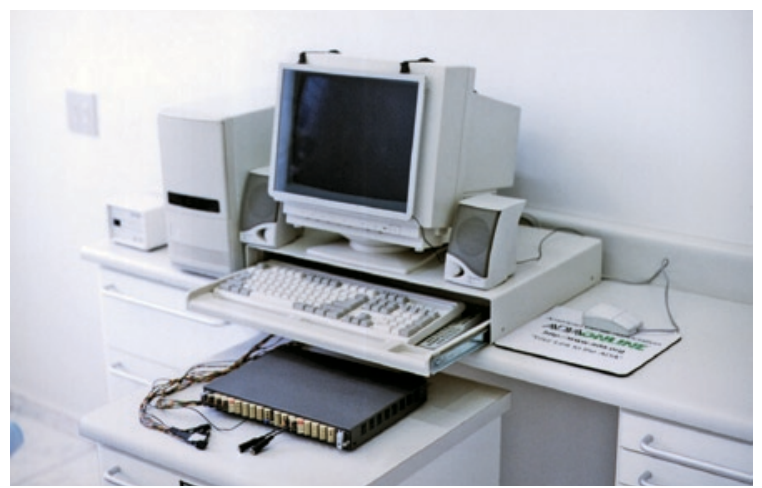

FIGURA 2 - B) Eletromiógrafo Lynx com sistema de Aquisição de Dados-placa CAD-12/32-Lynx. C) Computador de mesa com programas de tratamento e leitura de dados Aqdados-Lynx DOS/Windows.

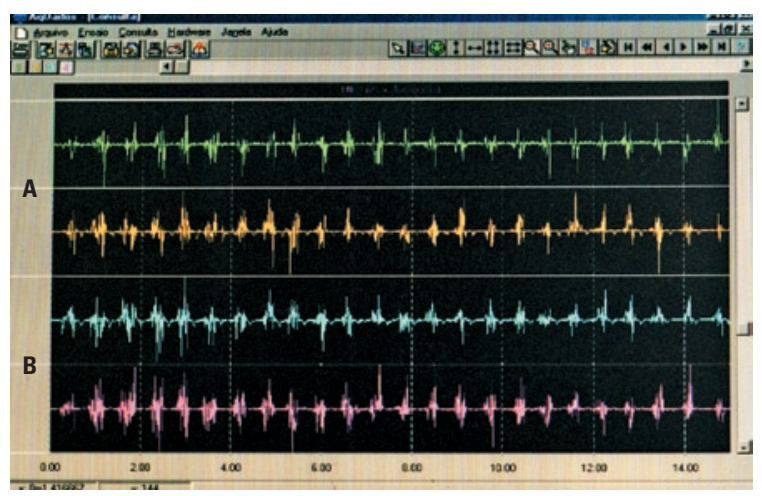

FIGURA 4 - Registros eletromiográficos durante os 10 segundos finais de mastigação habitual de parafilme (A) e cenoura (B), Os valores de RMS (Root Mean Square) ou Valor Eficaz são fornecidos diretamente pelo software. 
nâmica mandibular durante o exame eletromiográfico e os resultados, anotados em ficha clínica anexa. Após o registro de 20 ciclos mastigatórios consecutivos, considerou-se a presença de um lado de preferência mastigatória quando a mastigação ocorria com uma freqüência de ciclos mastigatórios pelo menos $30 \%$ maior por um dos lados ${ }^{12}$.

\section{RESULTADOS}

Após análise dos registros eletromiográficos durante mastigação habitual de cenoura foram obtidos os seguintes resultados estatísticos: verificou-se a freqüência de 20,7\% (6) de sujeitos com LPM direita, $62,1 \%$ (18) de sujeitos com LPM esquerda e 17,2\% (5) de sujeitos sem LPM (mastigação bilateral) conforme visto na tabela 1 e figura 5. No total, 24 dos 29 sujeitos da pesquisa possuíam um lado de preferência mastigatória durante mastigação habitual de cenoura, correspondendo a $82,8 \%$ da amostra.

Com a mastigação habitual de parafilme,

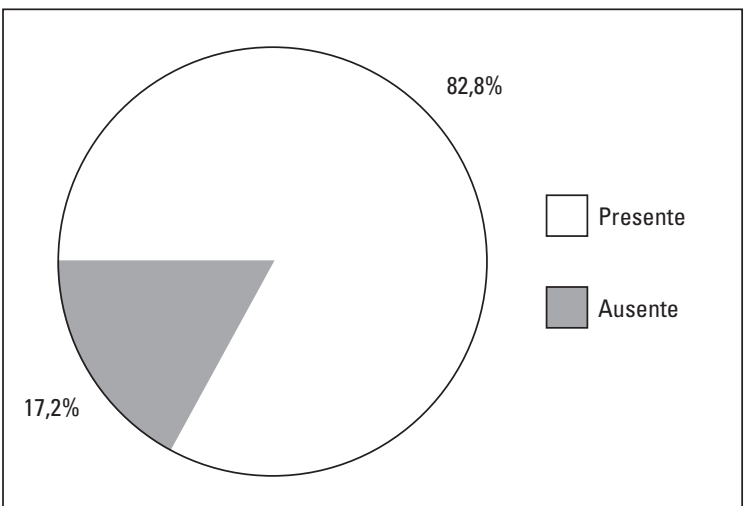

FIGURA 5 - Freqüência de um Lado de Preferência Mastigatória (LPM) após sua determinação através de registro eletromiográfico dos músculos masseteres direito e esquerdo durante mastigação habitual de cenoura.

Tabela 1 - Freqüência de um Lado de Preferência Masti-
gatória (LPM) após sua determinação através de registro
eletromiográfico dos músculos masseteres direito e
esquerdo durante mastigação habitual
de cenoura e parafilme.

verificou-se a freqüência de 5 sujeitos com LPM direita correspondendo a 17,2\% da amostra, 16 sujeitos com LPM esquerda $(55,2 \%$ da amostra) e 8 sujeitos sem LPM $(27,6 \%$ da amostra). No total, 21 dos 29 sujeitos da pesquisa possuíam um lado de preferência mastigatório durante mastigação habitual de parafilme, correspondendo a $72,4 \%$ da amostra. (Tab. 1, Fig. 6).

Houve concordância entre os resultados da análise eletromiográfica e as observações visuais em $83 \%$ dos sujeitos da pesquisa, quanto à verificação do lado de preferência mastigatória. Dos 29 sujeitos estudados, houve concordância em 24 sujeitos quando comparados os métodos de determinação da LPM, tanto para cenoura quanto para parafilme. (Tab. 2).

A média em RMS dos valores médios obtidos através do registro eletromiográfico dos músculos masseteres direito e esquerdo durante a mastigação habitual de cenoura e parafilme obtidos foram

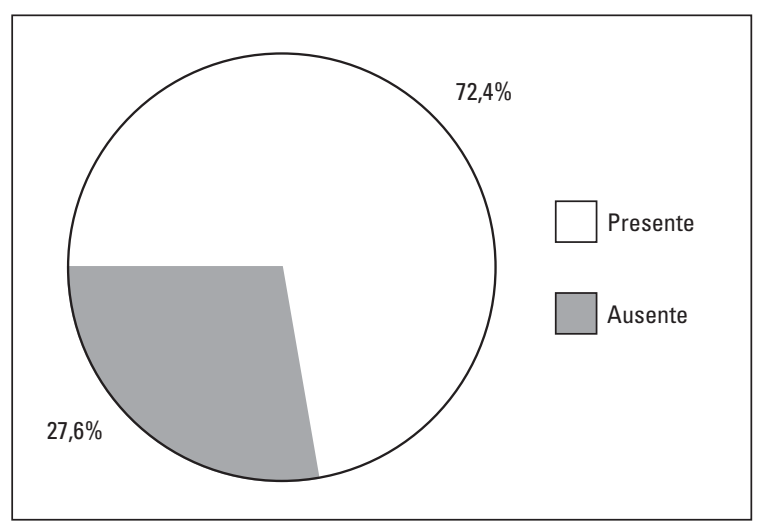

FIGURA 6 - Freqüência de um Lado de Preferência Mastigatória (LPM) após sua determinação através de registro eletromiográfico dos músculos masseteres direito e esquerdo durante mastigação habitual de parafilme.

Tabela 2 - Percentual de coincidência entre a verificação do lado de preferência mastigatória realizada através da observação visual direta e da eletromiografia dos músculos masséteres durante mastigação habitual de cenoura e parafilme.

Verificação do Lado de Preferência Mastigatória EMG Cenoura $x \quad$ EMg Parafilme $x$ Observação Direta Observação Direta

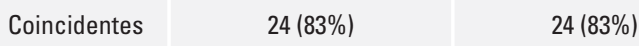

$\begin{array}{lll}\text { Divergentes } & 5(17 \%) & 5(17 \%)\end{array}$ 


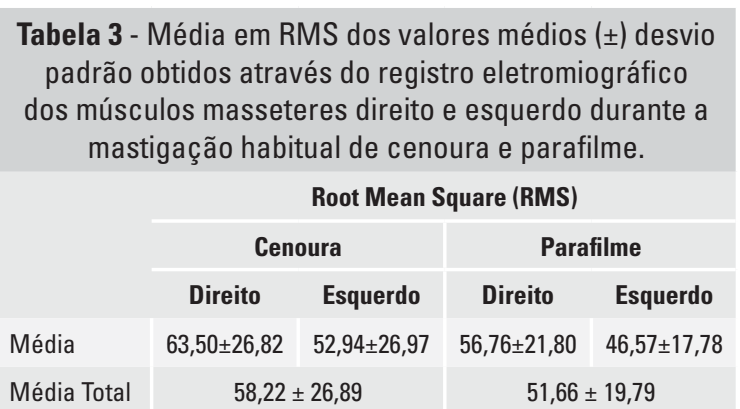

20\% maior do lado (esquerdo) durante mastigação de cenoura e $22 \%$ maior do lado esquerdo durante mastigação de parafilme (Tab. 3, Fig 7).

\section{DISCUSSÃO}

Os resultados obtidos neste estudo demonstraram a presença de um lado de preferência mastigatória na maioria dos sujeitos estudados durante mastigação habitual de cenoura $(82,8 \%)$ e parafilme $(72,4 \%)$ com uma média geral equivalente a $77,6 \%$ dos sujeitos com um lado de preferência mastigatória. Estes resultados estão de acordo com os resultados obtidos por Hoogmartens e Cauber$\mathrm{gh}^{7}$ onde $77,8 \%$ da amostra demonstraram possuir um lado de preferência mastigatória e com Ahlgren ${ }^{1}$ que, durante Simpósio sobre Aspectos Clínicos e Fisiológicos da Mastigação, salientou que via de regra, a maioria das pessoas de origem européia (civilizados, com alimentação industrializada) mastiga unilateralmente, enquanto que os indivíduos de origem mais primitiva mastigam bilateralmente, alternando, com bastante regularidade entre os lados direito e esquerdo. Vários pesquisadores verificaram a presença da mastigação bilateral alternada com movimentos mandibulares

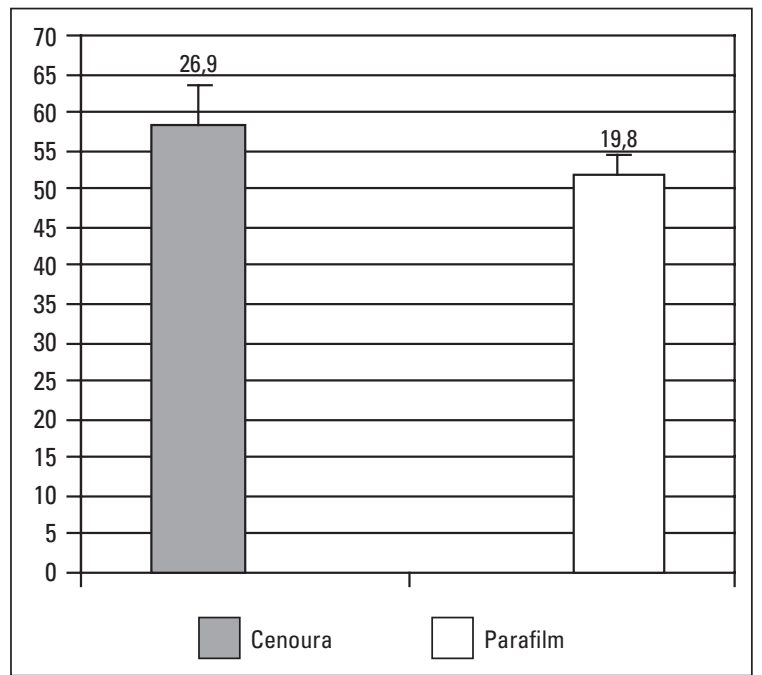

FIGURA 7 - Média dos valores médios individuais de RMS obtidos através de registro eletromiográfico dos músculos masseteres direito e esquerdo durante mastigação habitual de cenoura e parafilme.

realizados por ambos os lados, com grande amplitude e notável regularidade ${ }^{1,2,3,23}$.

A determinação do lado de preferência mastigatória a partir de valores de RMS 20\% maiores em relação ao lado de não preferência foi baseado na média das diferenças dos valores de RMS entre o lado direito e esquerdo dos sujeitos pesquisados. Apesar do parâmetro usado ter sido adotado dentro dos valores obtidos no experimento, a prevalência de um lado de preferência mastigatória verificada neste estudo $(77,6 \%)$ mostrou-se semelhante à prevalência de um lado mastigatório preferencial obtido por outros pesquisadores ainda que se utilizando metodologia diferente como, por exemplo, a contagem dos ciclos ao invés dos valores de contração muscular ${ }^{7,12}$.

Contudo, em relação à distribuição do Lado de Preferência Mastigatória (LPM), enquanto Hoogmartens e Caubergh ${ }^{7}$ verificaram um equilíbrio na distribuição do lado de preferência (com freqüência de LPM esquerda $=39,4 \%$ da amostra e LPM direita $=38,4 \%$ ) neste estudo verificou-se maior freqüência de LPM esquerdo (LPM esquerda em $62,1 \%$ e direita em $20,7 \%$ da amostra durante a 
mastigação de cenoura).

Durante a mastigação habitual de parafilme verificou-se LPM esquerdo em 55,2\% e LPM direito em $17,2 \%$ da amostra.

A ausência de um lado de preferência mastigatória verificada neste estudo foi observada em apenas $17,2 \%$ da amostra durante mastigação habitual de cenoura e $27,6 \%$ durante mastigação habitual de parafilme. Aparentemente esta diferença se deve ao fato de que a mastigação de parafilme ocorre com uma menor contração muscular. A cenoura exige mais força para ser fragmentada e triturada. Os valores médios de RMS obtidos durante a mastigação de parafilme foram 13,3\% menores que os valores obtidos durante mastigação de cenoura. A média obtida a partir dos valores médios de RMS de cada sujeito (3 exames para cada sujeito) durante a mastigação habitual de cenoura $(58,22 \pm 26,89)$ mostrarou-se menor que a média dos valores durante mastigação de parafilme $(51,66 \pm 19,79)$ como pode ser visto na tabela 2. Os valores altos para o desvio padrão indicariam a alta variação dos níveis de contração muscular individuais dependendo da potência muscular, eficiência mastigatória, além de outras características individuais ${ }^{10}$.

Verificou-se maior freqüência de um lado de preferência mastigatória durante mastigação habitual de cenoura $(82,8 \%)$ que de parafilme $(72,4 \%)$. Esta diferença pode estar relacionada com a fase de deslizamento dentário intimamente relacionada com a trituração dos alimentos ${ }^{20}$. Para que haja a trituração adequada do alimento e posterior deglutição a cenoura provoca um movimento mastigatório caracterizado por um ciclo mastigatório mais amplo que o do parafilme. Thexton ${ }^{22}$ questionou como os impulsos sensoriais interagiriam nos geradores de padrão rítmico do tipo considerado por Lund ${ }^{9}$. Afirmou que a mastigação é programada no sentido de que representaria os impulsos eferentes de circuitos neurais organizados, porém, excluindo-se os estágios iniciais da primeira mordida, está sujeita a modificações consideráveis por retroalimentação periférica.

O movimento de trituração tem como característica um ciclo mastigatório com uma extensão maior tanto no sentido vertical como horizontal com uma fase de deslizamento dentário mais extensa o que acaba por criar com o tempo de utilização constante de alimentos duros, facetas de desgaste características. Os alimentos menos consistentes requerem um movimento mais vertical com golpes cuspídicos mais cêntricos, com menos deslizamento dentário. Talvez esta seja a causa da diferença verificada neste trabalho quando ao lado de preferência mastigatória entre cenoura (mais fibroso) e o parafilme (menos consistente). Mais investigações devem ser realizadas para verificar se alimentos com texturas diferentes e principalmente analisando os registros eletromiográficos feitos simultaneamente com registros cinesiográficos, mudariam o padrão mastigatório de preferência.

O tempo de 10 segundos determinado para os registros de mastigação habitual possibilita em média de 18 a 20 ciclos mastigatórios. Os valores de RMS são obtidos a partir da somatória de todos os ciclos. A mastigação preferencialmente unilateral é aquela onde pelo menos 30\% dos ciclos ocorre de um lado $^{12}$. A maioria dos estudos registra a mastigação deliberada e com um número de ciclos pré-determinados determinando maiores diferenças entre os EMG direito e esquerdo. Durante a mastigação habitual, ocasionalmente ocorrem mudanças de lado de mastigação. Portanto valores em torno de $20-25 \%$ já caracterizam a mastigação preferencialmente unilateral. Estes dados foram confirmados pela análise visual mostrando uma coincidência de $83 \%$ entre os métodos de verificação. Christensen e Radue ${ }^{4}$ verificaram uma coincidência de $78 \%$ entre as observações visuais e a os registros eletromiográficos.

Deve-se salientar que a mastigação habitual não é controlada pelo córtex motor como a mastigação voluntária e sim por núcleos extrapiramidais caracterizando-se por ser inconsciente. 
Pode-se concluir que a maioria dos sujeitos examinada nessa pesquisa possuía um lado de preferência mastigatória durante mastigação habitual de cenoura $(82,8 \%)$ e parafilme $(72,4 \%)$ e que os métodos eletromiográfico e visual podem ser utilizados para a determinação da existência de um lado de preferência mastigatória.

\section{AGRADECIMENTOS}

Agradecimentos à Capes (Coordenação de Aperfeiçoamento de Pessoal de Nivel Superior), pelo suporte financeiro à pesquisa (DS-44/97). Um agradecimento especial à Profa. Regina Maria Puppin Rontani pela orientação na correção do trabalho e ao Prof. Fausto Bérzin pela importante contribuição na coleta dos dados.

Enviado em: Setembro de 2003

Revisado e aceito: Outubro de 2003

\title{
Masticatory preference side detection using electromyography exam compared to the visual analysis
}

\begin{abstract}
The objective of this study was to verify the frequency of masticatory preferential side using electromyography exam compared to the visual inspection. The sample was comprised by 29 healthy students aged 18 to 25 years, of both sexes, from Araras School of Dentistry-UNIARARAS, Brazil, selected following approach: complete permanent dentition, except third molars, with no clinical signs of the temporomandibular dysfunction or periodontal disease. The electromyography analysis was accomplished in the right and left masseter muscles during habitual mastication of carrot and Parafilm. It was considered as presence on a masticatory preferential side and unilateral mastication when the muscular contraction width values obtained in RMS (Root Mean Square) has had at least $20 \%$ as a difference between the right and left masseter muscles. The visual inspection was carried out during the electromyography data acquisitions by only one observer. He determined the masticatory preferential side taking account the number of masticatory cycles developed by each side. The masticatory preferential side was considered when 30\% of the cycles were developed in only one side after the registration of 20 consecutive masticatory cycles. Of the total sample, 82.8\% (24/29) and 72.4\% (21/29) exhibited a preferential masticatory side during habitual carrot and Parafilm mastication, respectively. The concordance level between the electromyography and visual inspection exams was $83 \%$ of the total observations. It could be concluded that most of the subjects examined in this study exhibited a masticatory preferential side and that the electromyography can be used as a parameter for the masticatory side detection.
\end{abstract}

Key words: Mastication. Masticatory preference side. Unilateral mastication. Electromyography. 


\section{REFERÊNCIAS}

1. AHLGREN, J. Movimentos mastigatórios no homem. In: ANDERSON, D.J.; MATHEWS, B. Mastigação. 1. ed. Rio de Janeiro: Guanabara Koogan, 1982.

2. BARRET, M. J. Masticatory and non-masticatory use of Teeth. In: WRITH, R. V. S. Stone tools as culture markers: change, evolution and complexity. Canberra: Australian Institute of Aboriginals Studies, 1977. cap.1, p.18-23.

3. Beyron, H. L. Occlusal relations and mastication in Australian aborigines. Acta Odontol Scand, Oslo, v. 22, p. 597-678, 1964.

4. CHRISTENSEN, L.V, RADUE, J.T. Lateral preference in mastication: an electromyographic study. J Oral Rehabil, Oxford, v.12, no. 5, p. 429-434, Sept.1985.

5. HILDEBRAND, G. Y. Studies in the mastigatory moviments of the lower jaw. Scand Arch Phisyol, [S.I.], v. 61, p. 22-33, Jan. 1931.

6. HOOGMARTENS, M. J.; CAUBERGH, M.A. Chewing side preference during the first chewing cycle as a new type of lateral preference in man. Electromyogr. Clin Neurophysiol, Limerick, v. 27 , no. 1, p. 3-6, Feb.1987

7. HOOGMARTENS, M. J.; CAUBERGH, M. A. Chewing side preference during the first chewing cycle as a new type of lateral preference in man. Electromyogr Clin Neurophysiol, Limerick, v. 27, no.1, p. 3-6, Feb.1987.

8. KAZAZOGLU, E.; HEATH, M. R.; MULLER, F. A simple test for determination of the preferred chewing side. J Oral Rehabil, Oxford, v.21, no. 6, p.723-725, Nov.1994.

9. LUND, J. P. Evidências de um gerador de Padrão Neural Central regulando o Ciclo Mastigatório. In: ANDERSON, D. J.; MATHEWS, B. Mastigação. 1. ed. Rio de Janeiro: Guanabara Koogan, 1982.

10. MOHAMED, S. E.; CHRISTENSEN, L.V.; HARRINSON, J. D. Tooth contact patterns and contractile activity of the elevator jaw muscles during mastication of two different types of food. J Oral Rehabilit, Oxford, v.10, p. 87-95, 1983.

11. MOLLER, E. Action of the muscles of mastication. Front Oral Physiol, Basel, v.1, p.121-58, 1974.

12. MONGINI, F. ATM e músculos craniofaciais: fisiopatologia e tratamento. São Paulo: Ed. Santos, 1998. cap. 7.
13. PIGNATARO NETO, G. Análise da correlação entre os ângulos funcionais mastigatórios direito e esquerdo com o lado de preferência mastigatória. 2000. 98 f.Dissertação (Mestrado em Odontologia)- Faculdade de Odontologia de Piracicaba, Universidade Estadual de Campinas, Piracicaba, 2000.

14. PLANAS, P. Rehabilitación Neuro-oclusal (RNO). 2. ed. Madrid: Ed. Barcelona: Edit. Científicas y Técnicas, 1994. cap. 3.

15. POND, L. H.; BARGHI, N.; BARNWELL, G. M. Occlusion and chewing side preference. J Prosthet Dent, St. Louis, v. 55, no. 4, p. 498-500, Apr.1986.

16. POYKELA, A. et al. Effect of unilateral masticatory function on craniofacial growth in the rabbits. Eur J Oral Sci, Copenhagen, v.103, no. 2, p.106-111, Apr.1995.

17. POYKELA, A.; KANTOMAA, T.; PIRTTINIEMI, P. Craniofacial growth after a period of unilateral masticatory function in young rabbits. Eur J Oral Sci, Copenhagen, v. 105, no. 4, p. 331-337, Aug.1997.

18. RAMFJORD, S. P.; ASH, M. M. Oclusão. 3. ed. Rio de Janeiro: Interamericana, Tradução: Dioracy F. Vieira, 1984.

19. RIISE, C.; SHEIKHOLESLAM, A. Influence of experimental interfering occlusal contacts on the activity of the anterior temporal and masseter muscles during submaximal and maximal bite in the intercuspal position. J Oral Rehabil, Oxford, v. 10, no. 3, p. 207-214, May 1983.

20. RILO, B. et al. Frontal-plane lateral border movements and chewing cycle characteristics. J Oral Rehabil, Oxford, v. 28, no.10, p. 930-936, Oct. 2001.

21. SIMÕES, W.A. Visão do crescimento mandibular e maxilar. JBO, Curitiba, v. 3, no.15, p. 9-18, maio/jun.1998.

22. THEXTON, A. J. Até que ponto a mastigação é programada e independente de retroalimentação periférica? In: ANDERSON, D. J.; MATHEWS, B. Mastigação. 1. ed. Rio de Janeiro: Guanabara Koogan,1982.

23. VAN DER LAAN, T. Função mastigatória em índios lanomami. 1998. 68 f. Dissertação (Mestrado em Ciências)-Faculdade de Odontologia de Piracicaba, Universidade Estadual de Campinas, Piracicaba, 1998.
Endereço para correspondência

Godofredo Pignataro Neto

IBRAFO - Instituto Brasileiro de Fisiologia Oral

Rua Padre Anchieta, 15

Belvedere

CEP: 13601-130

Araras - SP

E-mail: pignataroneto@internetsp.com.br pignataroneto@ibrafo.org.br

Site: www.ibrafo.org.br 